\title{
Some Properties of the Electromagnetic Radiation Emitted by the HV Transformers
}

\author{
Nikolay V. Kinsht, Natalia N. Petrun'ko, Peter V. Katz \\ Tech. Diagnosis Lab. Institute of Automation \& Control Processes FEB RAS, Vladivostok, Russia \\ Email: kin@dvo.ru
}

Received April, 2013

\begin{abstract}
One important way to control the technical condition of the high-voltage power equipment element is the monitoring and interpretation of electromagnetic radiation. Analysis of the spectra of emitted high-voltage equipment EMR provides information on which determines the intensity discharge processes occurring in insulation and other structural elements of high voltage equipment under the operating voltage without interfering with the process.
\end{abstract}

Keywords: Partial Discharge; Power Transformer; Emitted Electromagnetic Radiation; Technical Condition

\section{Partial Discharges in the HV Devices Insulation}

Development of new methods of diagnostics is today one of the most science-intensive areas of research and assessment of the technical condition and residual resource of high-voltage transformer equipment (TE) in the early stages of degradation of its technical conditions (TC). One of the reasons for the lack of effectiveness of the methods of TE early diagnostics is the lag in the fundamental component of comprehension of electrophysical processes occurring in the real objects.

Technical conditions of the TE are largely determined by the condition of its insulation. Partial electrical discharges (PD) arise as during normal operation of the equipment, as when the degradation of the insulation and other structural elements. PD is the primary electrophysical process, and they characterize the equipment quality.

According the International Electrotechnical Commission (IEC) International Standard 60270 - 2000 [1] the principles of the method, which is applied to the present time and based on the concept of so-called "apparent charge" well known. They are formulated over 70 years ago. The simplest mathematical model based on "apparent charge" is constructed on the analysis of the discharge in the single inclusion in the insulation. However, the practice of operation of the responsible power equipment showed that the means of control of the TCS of the equipment, based on the concept of apparent charge, could not prevent large-scale accidents.

Our researches [2] has shown that this concept is rather poor, it does not take into account a number of essential factors: the geometric arrangement of inclusion in the space of insulation, the variation of the ignition voltage of the $\mathrm{PD}$, the uncertainties in the voltage of extinction of the PD, the duration of the burning of the PD, insulation $\operatorname{tg} \delta$. These factors are the parameters of a common electrophysical processes; in the aggregate variation of these factors is the uncertainty in several orders of magnitude. In addition, this concept is fundamentally not taking into account the collective effects of the large number PD. Some ways time-frequency representation of a set PD as, for example, it is represented in Figure 1 are known. However such analysis is insufficient for practice.

\section{EMR as a Diagnostic Sign}

PD are caused own electromagnetic radiation (EMR) in a wide range of frequencies, which can be clearly detected

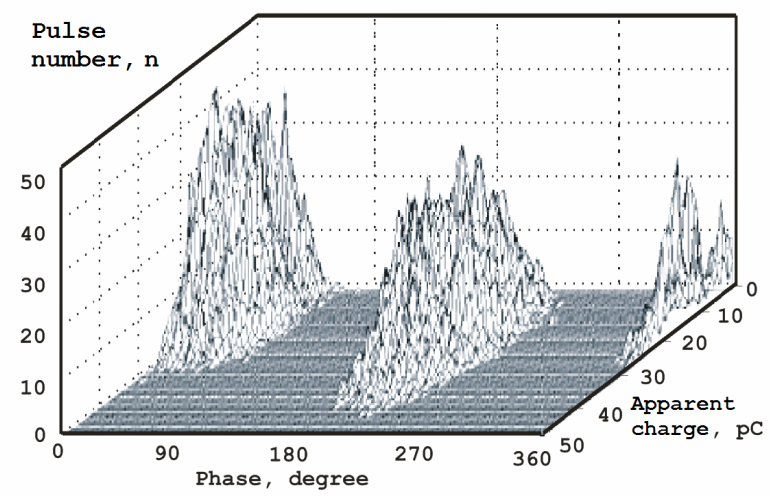

Figure 1. Example of a phase-numbers representation of 'apparent' charges. 
in operation mode, and recorded the EMR spectral characteristics with the radio-electronic equipment [3-5]. The results of the simulation of the radioactive properties of the entry are confirmed by research on real objects, have allowed to formulate a methodology for the monitoring of the spectra of EMR; protected by several Russian patents.

No recommendations are given in the Great Britain standard for either measuring methods or bandwidth/ frequencies of instruments to be used in such investigations, as these methods or instruments, in general, do not directly quantify the apparent charge of PD current pulses [6]

The idea of registration of EMR radiated by the HV equipment, in it, is elementary, on this basis were built sensors of the PD, located directly on the equipment.

The problem consists only in the fact, what kind of measuring equipment is used for the registration EMR and how the results are interpreted. At the present time among the experts there is no single opinion on the properties of the EMR emitted TE. So, according to some data, the study of the TE's EMR does not make sense to produce in the range above $25-40 \mathrm{MHz}$. According to some researchers, EMR entry is advisable to register in the range of $1-3 \mathrm{GHz}$, and the recommendations of the well-known company Doble, proposing to use in practice analyzer of the $\mathrm{PD}$, are in the monitoring of the PD in the range of $250-1000 \mathrm{MHz}$ [7].

On the experimental data of the authors the information component of EMR TEs is not evenly distributed from a few megahertz to a few gigahertzes. Since the time of the PD current is estimated as $10^{-8} \ldots 10^{-9} \mathrm{~s}$, and, therefore, the upper part of the range of frequencies of natural TE's EMR extends down to the centimeter waves. The question consists only in search of information frequency bands EMR and effective selection of the signals possessing the greatest informative and noise immunity from the point of view of diagnostics of a technical condition TE.

Study of this issue is of great importance in connection with the appointment of the technical parameters of the measuring set, controlling the operation of the TE and the use of appropriate algorithms of information processing and decision-making. From the point of view of the theory of random processes posterior integral implementation of the EMR, as well as their TE's spectra are non-stationary random processes, which is modulated of the frequency operating voltage. It is evident, however, that in them there is a clearly expressed the informational component; these spectra are specific to individual elements of TE and are similar to the same type of EA.

In Figure 2 are given fragments of 3 the same type single-phase power transformers $167000 \mathrm{~kW} \mathrm{500/220} \mathrm{kV}$

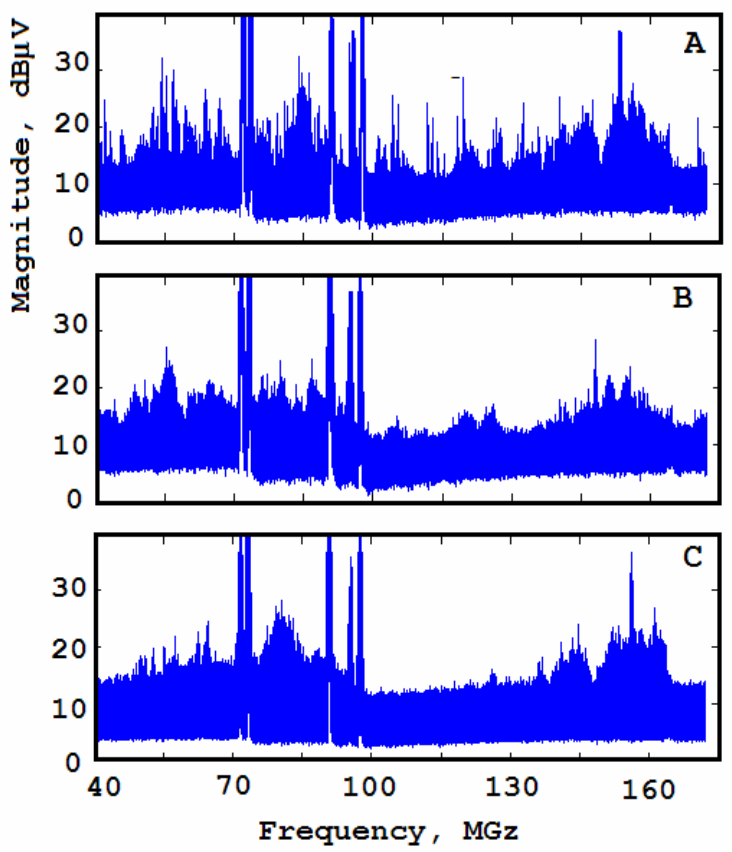

Figure 2. Example of a spectra EMR of the same type transformers.

in the range $40-170 \mathrm{MHz}$ in the Zeya hydroelectric power station. Stable explicit spectral lines correspond to the TV channels.

\section{Time-frequency Characteristics of the EMR Emitted from TE}

Spectrum observed visually on the screen of a spectrum analyzer, is a very dynamic process. Single partial discharges in the insulation TE excite the electromagnetic oscillations in a wide frequency range inside the transformer tank.

Transformer at high frequencies may be represent as a difficult equivalent circuit, the elements of its construction-winding, magnetic core, insulation in the aggregate have a resonance properties of high quality, the frequency response has a lot of resonances (natural frequencies). The energy of the PD excites the transformer rather lengthy intrinsic high-frequency electromagnetic oscillations, and emits them in the frequency ranges relevant to natural frequencies. Integrally this information can be represented in the graphical form as a three dimensional form as showed on Figure 3.

In the simplified approach, the spectra are measured their maximum, minimum and average value of signal amplitude (as on separate frequencies, so in a certain range), as well as the root mean square deviation and the energy spectrum. However, significantly more effective appears to be more detailed time-frequency analysis. Identification of frequency bands and amplitudes of these oscillations is the main task of the study. 


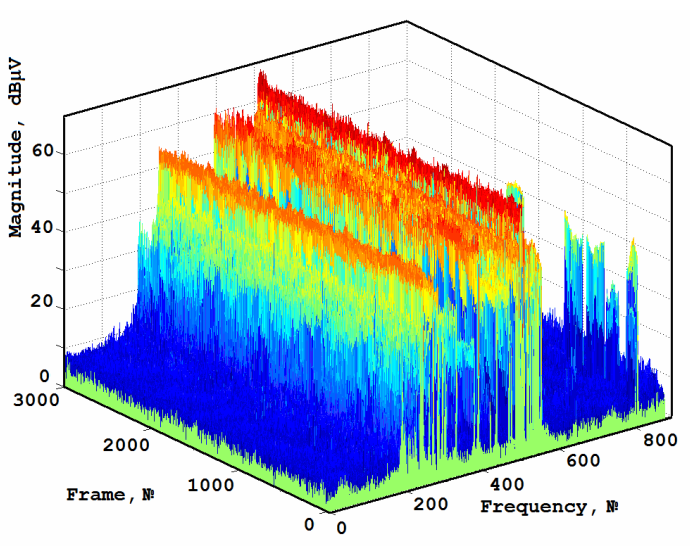

Figure 3. Example of amplitude spectra of EMR.

\section{The Interpretation of the Experimental Spectra}

The subsequent presentation illustrates typical data from the archives of the results of inspections of the power transformer equipment on the $500 / 220 \mathrm{kV}$ substation in Siberia and the Far East Russia, which were made at the Laboratory for Electrophysics and Electric Power IACP. (Provided data correspond to the range of frequencies 40 ... $170 \mathrm{MHz}$. Further concrete values of frequencies and time points don't represent special interest; they will be identified only on the serial numbers appropriated at processing of results).

Considering the maximum spectra, we note quite a number of local maxima. These peaks may correspond to both EMF initiated PD, and may correspond to other sources of electromagnetic radiation, which is an obstacle to the technology in its own analysis of the equipment EMR. These other sources, on the one hand, (in turn) may be the information signals of various kinds (eg, radio and TV) and on the other hand, they may be the result of industrial pollution of air. Clearly, the more reliably able to identify local maxima of the spectrum corresponding to the $\mathrm{PD}$, the results would be more correct assessment of the technical condition of the equipment. Here there are two factors that must be taken into account. So, first, for diagnostics it is necessary not only to determine the characteristic frequencies of own equipment EMR. Taking into account possible variations in the parameters, the actual paths of radiation quality factor, inaccurate calculations and natural measurement errors need to associate each characteristic frequency sub-band electromagnetic radiation to be monitored. Second, it may be that even when correctly and reliably identified above "useful" sub bands EMR noises significantly exceed the level of "useful" signal. Thus the observation of electromagnetic radiation in the sub-band will give false information. Ultimately, the choice for reliable "workers" subbands informative in terms of monitoring partial discharges must be combined with a time frequency analysis.

Let's show the stated principles and problems on examples.

Let's consider the maximum EMR spectrum registered at the transformer $125000 \mathrm{~kW} 220 / 110 \mathrm{kV}$ (Figure 4 corresponding given above 3 -d spectrum). Let's imagine that there are bases to look for information subband in the range of frequencies with numbers $[410 \ldots 460]$. If to consider an amplitude range is more detailed, in this interval there will be 2 maxima, with numbers about 430 and 441-442, respectively (Figure 5) which can be potential considered as subband of the transformer EMR.

A fragment of the full $3 \mathrm{~d}$ time-frequency spectrum corresponding to the frequency range under consideration makes it possible to clarify this issue (Figure 6).

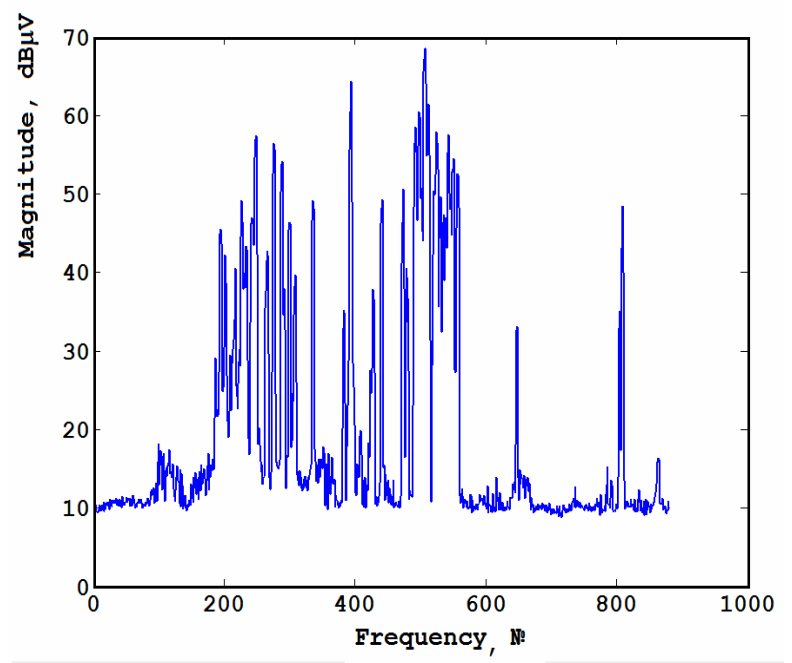

Figure 4. Maximum EMR spectrum of the transformer $125000 \mathrm{~kW} \mathrm{220/110} \mathrm{kV.}$

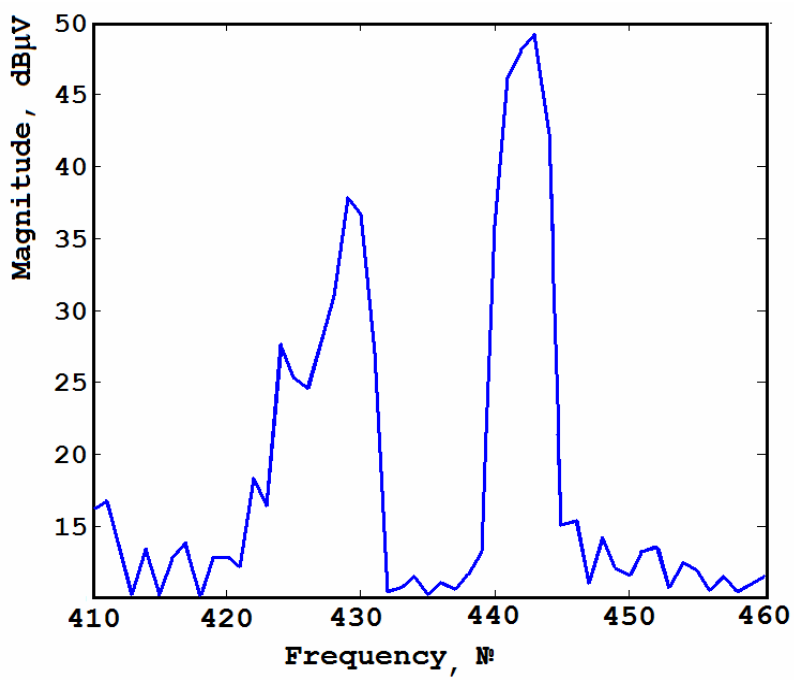

Figure 5. The fragment of the Figure 4 spectrum. 
Thus, the time dependence of the signal at the frequency of EMR № 441 shows that this signal is typical for TV (Figure 7) and the time dependence of the signal at the frequency of EMR № 429 (Figure 8) is clearly associated with partial discharges.

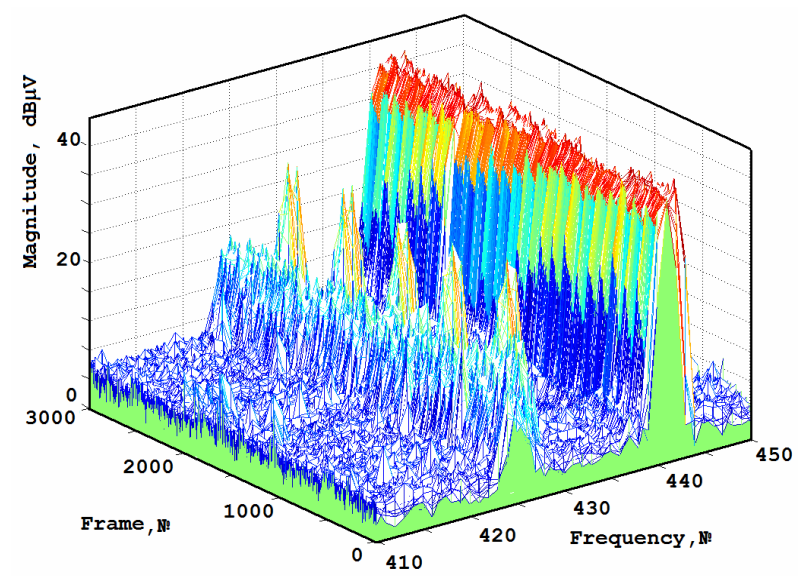

Figure 6. The fragment of the full 3d time-frequency.

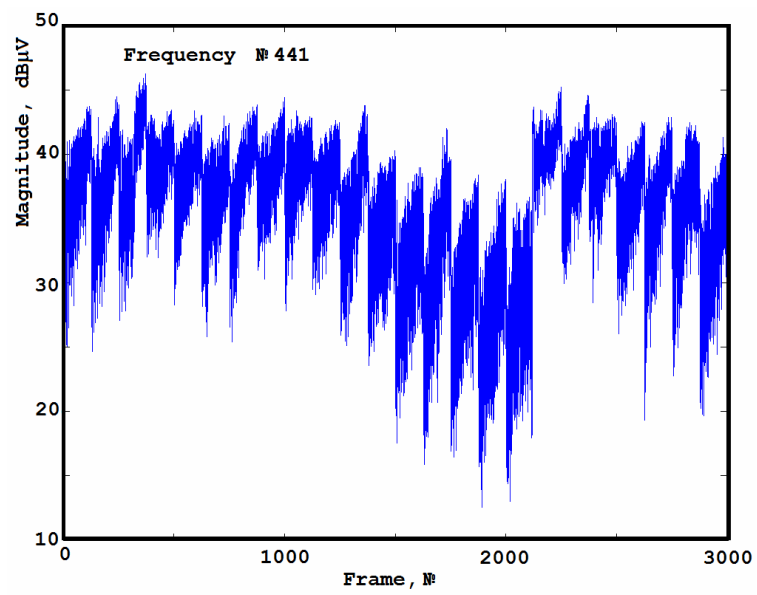

Figure 7. The signal at the frequency of EMR № 441.

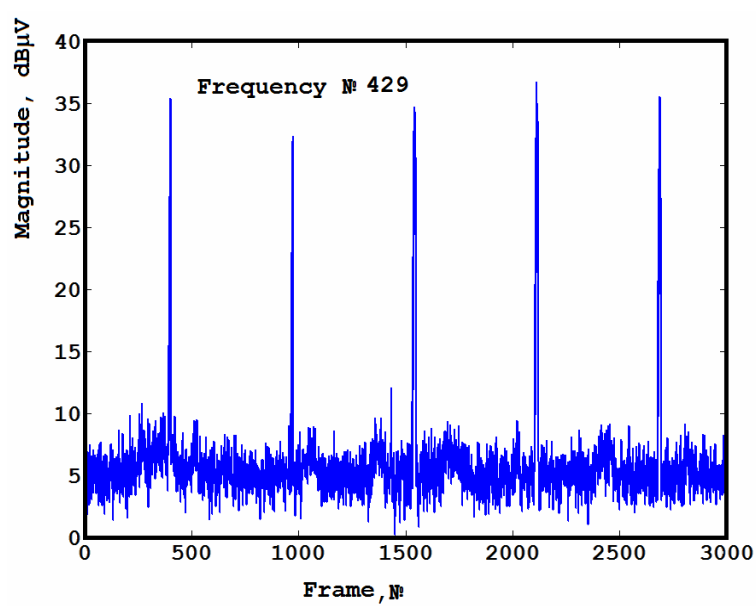

Figure 8. The signal at the frequency № 429.

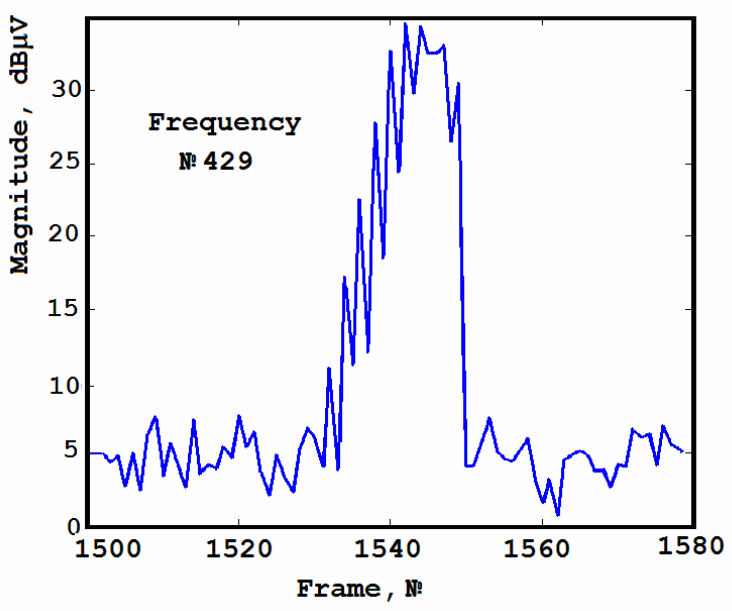

Figure 9. The fragment of the signal at the frequency of EMR № 429.

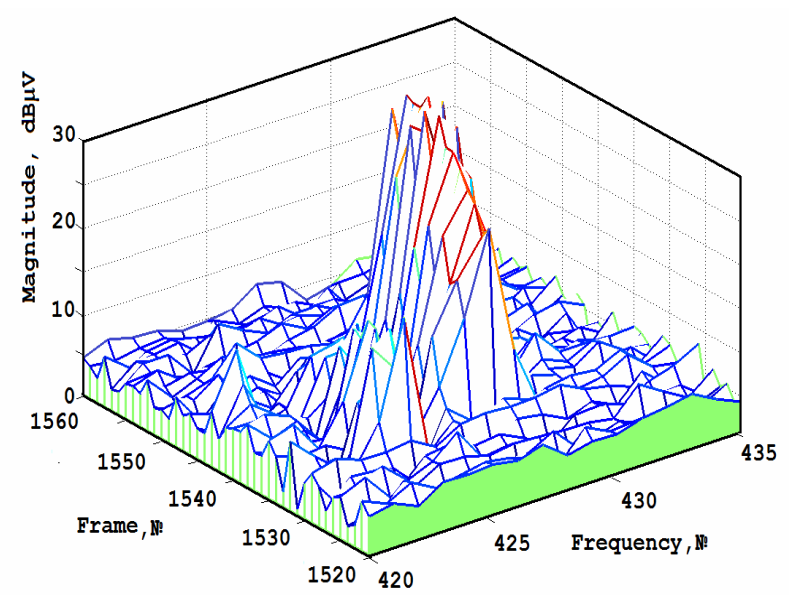

Figure 10. The fragment of the 3d time-frequency spectrum nearby frequency № 429 and frame № 1540.

Moreover, having analyzed the impulse which has arisen in the range of time, corresponding $1500 \ldots$ to 1580 frames, we can say it is a series of more than 10 partial discharges (Figure 9). Finally, the most obvious is the registered pulse $3 \mathrm{~d}$ representation EMR reflecting a series of partial discharge, which is clearly seen its localization in both time and frequency (Figure 10). The degree of localization is due to the quality factor of this natural frequency.

\section{Conclusions}

To study the elements of EMR near high-voltage equipment is to identify criteria that characterize the condition of the internal technical equipment. The most informative is the analysis of time-frequency properties of their own EMR spectra of the equipment.

The frequencies corresponding to the individual lines of EMR related to partial discharges are the natural 
frequencies of the transformer.

\section{Acknowledgements}

This work was supported by Russian Foundation for Basic Research (grant N13-08-00924)

\section{REFERENCES}

[1] International IEC Standard 60270-2000.

[2] N. V. Kinsht and M. A. Katz, "Some Problems of the Partial Discharge Burning Time," TEEM, Vol. 7, No. 6, 2006, pp. 319-323.

[3] N. V. Kinsht and N. N. Petrun'ko, "The Experience of
Inspection of a Technical Condition of the HV Equipment on Substations by a Method of Registration of the Own Electromagnetic Radiations," in Proceedings of CMD2008, 2008, pp.738-740.

[4] N. V. Kinsht and N. N. Petrun'ko, "Research on Characteristics of EMR of the High-Voltage Equipment during the Process of its Diagnostic," in Proceedings of CMD2010, 2010, pp. 1149-1152.

[5] N. V. Kinsht and N. N. Petrun'ko, "Interpretation of Monitoring Dataof Own Electromagnetic Radiation HV Transformers" in Proceedings of CMD2012, 2012, pp 569-572.http://www.samgor.com/pdf/IEC60270.pdf.

[6] http://www.doble.com/litlib/files.html/download/395 